\title{
Case report: two rare cases of mixed epithelial and stromal tumor of the kidney and a review of the literature
}

\author{
Huangwei Huang ${ }^{1}$, Xuewen Jiang ${ }^{2}$, Benkang Shi ${ }^{2}$, Gang Yin ${ }^{2}$, Jianfeng Cui ${ }^{1}$, Changsheng Guo ${ }^{3}$, \\ Chunxiao Yu ${ }^{4}$, Hui Meng ${ }^{2}$ \\ ${ }^{1}$ Department of Urology, Cheeloo College of Medicine, Shandong University, Jinan, China; ${ }^{2}$ Department of Urology, Qilu Hospital of Shandong \\ University, Jinan, China; ${ }^{3}$ Department of Urology, Traditional Chinese Medicine Hospital of Leling City, Leling, China; ${ }^{4}$ Department of Urology, \\ Central Hospital of Zaozhuang Mining Group, Zaozhuang, China \\ Correspondence to: Hui Meng. Department of Urology, Qilu Hospital of Shandong University, Jinan 250012, China. Email: menghui0504@163.com.
}

\begin{abstract}
Mixed epithelial and stromal tumor of the kidney (MESTK) is rare renal neoplasm, which usually behaves benignly, while very rarely malignancies have also been reported. Histologically, MESTK is composed of both mesenchymal and epithelial components, where the epithelial components are arranged in a tubular or tubule-cystic pattern against a background of ovarian-like stromal proliferation. MESTK is more commonly seen in perimenopausal women or in patients on long-term estrogen replacement therapy. Given the popularity of routine health screening, patients main present asymptomatically. We report one rare case of MESTK, which was diagnosed in a 30-year-old woman. A computed tomography (CT) scan revealed one well-defined, uneven mass in the left kidney. The tissue obtained by fine-needle aspiration showed relatively homogeneous cells. Renal cell carcinoma could not be excluded, and left complete nephrectomy was performed, according to the patient's wishes. Another case of MESTK we present here was diagnosed in an 18-year-old male adolescent, who did not have a history of estrogen treatment, with estrogen treatment seen rarely in the clinical setting. Renal cell carcinoma was suspected, and a left partial nephrectomy was performed. Based on histopathological examination, the diagnosis was MESTK for both cases. Both patients were periodically monitored for one year following surgery and showed no imaging findings of recurrence or metastases. MESTK is benign tumor, and hence preoperative diagnosis is crucial to avoid overtreatment. To improve the current understanding of this disease, comprehensive studies on their pathogenesis and preoperative diagnosis are needed.
\end{abstract}

Keywords: Mixed epithelial and stromal tumors of the kidney (MESTK); kidney; computed tomography (CT); nephrectomy; case report

Submitted Feb 18, 2021. Accepted for publication Jul 05, 2021.

doi: $10.21037 /$ tcr-21-308

View this article at: https://dx.doi.org/10.21037/tcr-21-308

\section{Introduction}

Mixed epithelial and stromal tumors of the kidney (MESTK) is rare renal tumor, which usually behaves benignly, while very rarely malignancies have also been reported. Michal and Syrucek first defined MESTK in 1998 (1). Until 2020, about 200 cases of MESTK have been reported in the literature. Mixed epithelial and stromal tumors of the kidney is more commonly seen in perimenopausal women or patients on long-term estrogen replacement therapy. Cases in males are rarely reported, especially in teenagers. The female to male ratio is about $6: 1$, with the mean age being 46 years (2). Patients mainly present asymptomatically, but it is also possible that they present with flank pain, palpable abdominal mass, and hematuria. Computed tomography (CT) is relied on as a diagnostic imaging tool for MESTK. However, the final diagnosis depends on the histological features of the tumor and immunohistochemical (IHC) staining. The amount of preoperative fine-needle-aspirated tissue of renal cystic lesions is too little to make an accurate 

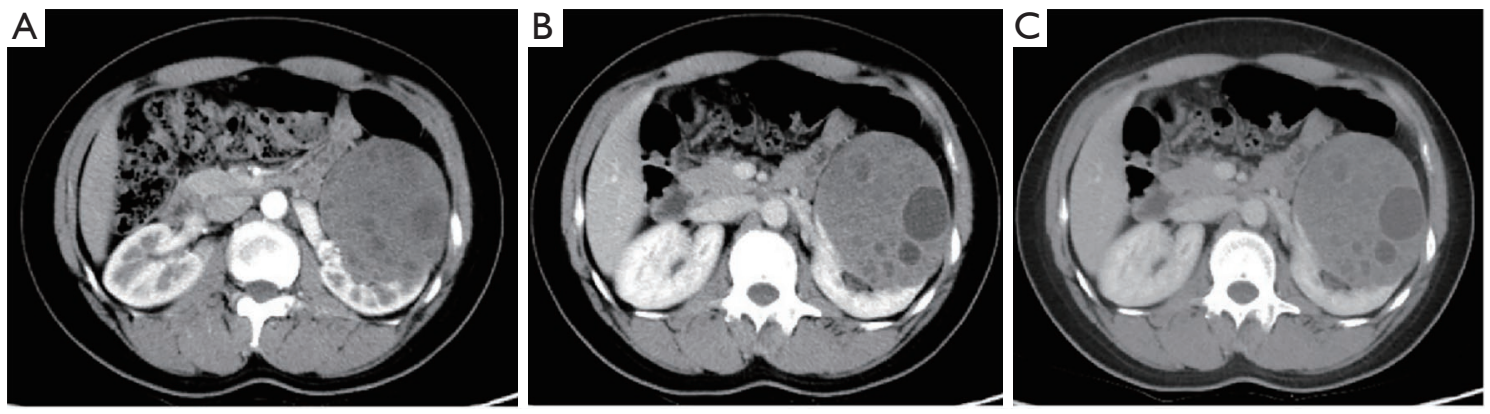

Figure 1 Abdominal computed tomography showed that the tumor had heterogeneous soft tissue. (A) In arterial phase; (B) in venous phase; (C) in secretory phase.

diagnosis. Because of the risk of malignant cell expansion, fine needle aspiration is not recommended in renal cystic lesions.

The present study reports two special cases of MESTK. One rare case is a 30 -year-old woman who underwent preoperative fine-needle aspiration, and the tissue obtained by fine-needle aspiration suggested a benign tumor, which, however, was consistent with the final histopathological diagnosis. Another case of MESTK we present here was diagnosed in an 18-year-old male adolescent, who did not have a history of estrogen treatment, with estrogen treatment being seen rarely seen in clinical setting. We also review previously published reports related to MESTK. Written informed consent was obtained from the patient.

\section{Case presentation}

\section{Case one}

A 30-year-old female was incidentally identified during a routine physical examination as having a mass on the left kidney; she did not have abdominal pain, hematuria, or weight loss. She did not report a history of estrogen treatment or significant medical history. Her family history was unremarkable. A physical examination using palpation was completely normal. Her routine blood investigations showed that hemoglobin (HGB) was $92.0 \mathrm{~g} / \mathrm{L}$, mean corpuscular volume (MCV) was $75.2 \mathrm{fL}$, and mean corpuscular hemoglobin concentration (MCHC) was $283.0 \mathrm{~g} / \mathrm{L}$, with the latter caused by irregular menstruation. Her other blood tests were within normal limits. The abdominal contrast-enhanced CT showed a $9.2 \mathrm{~cm} \times 7.3 \mathrm{~cm}$ well-defined, uneven mass lesion of the left kidney. The solid component showed mild to moderate enhancement which increased over time (Figure 1). The tissue obtained by CT-guided fine-needle aspiration showed relatively homogeneous cells without significant cytological atypia or mitosis. However, there was too little tissue to make an accurate diagnosis. The patient finally decided to undergo a left complete nephrectomy after the treatment of anemia. Macroscopically, the tumor measured $10 \mathrm{~cm} \times 9 \mathrm{~cm}$ and was close to the pelvis. Both multiloculated cystic structures and solid components were revealed in the tumor cross-section. Subsequent surgical pathological examination confirmed MESTK.

Microscopically, the tumor is composed of both mesenchymal components and epithelial components, and the epithelial components are arranged in a tubular pattern against a background of ovarian-like stromal proliferation, which in turn is composed of spindle cells (Figure 2). Immunohistochemically, the epithelial cells revealed positive expression of PAX-8 and CK. Some epithelial cells also revealed positive expression of GATA-3. The stroma of tumor showed positive expression of SMA and vimentin. There was no expression of CD10 and WT-1. The proportion of cells that expressed Ki-67 was less than $1 \%$. The patient was discharged without adverse outcomes. One month after the operation, no surgical complications were observed. The patient was periodically monitored for one year following surgery, and there were no imaging findings of recurrence or metastasis.

\section{Case two}

An 18-year-old male adolescent was incidentally identified with a mass on the left kidney during a routine physical examination; he did not report abdominal pain, hematuria, or weight loss. He did not have a history of estrogen treatment or significant medical history. Physical examination by 

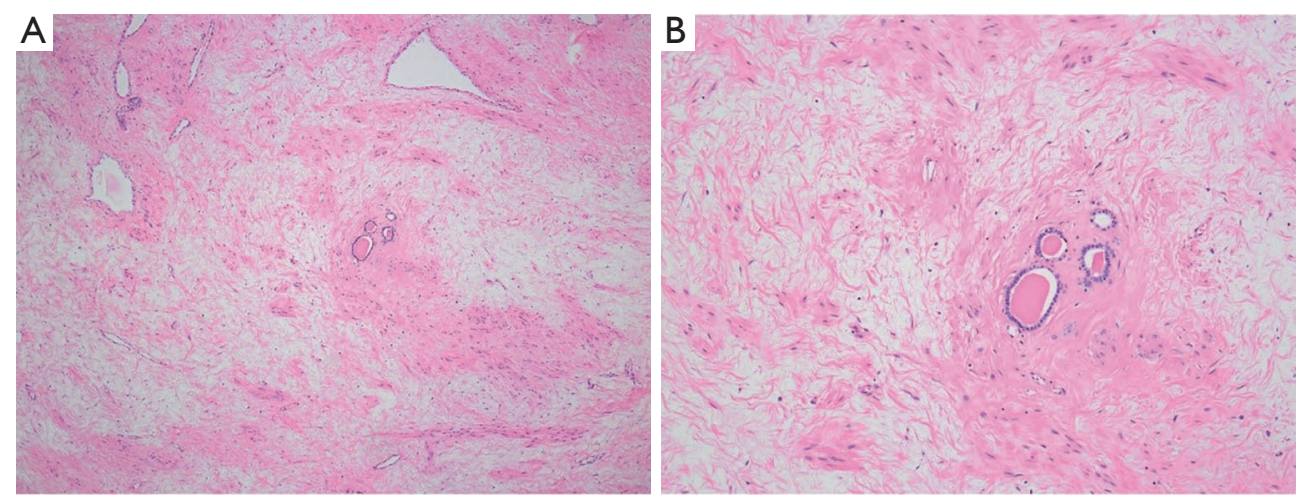

Figure 2 Microscopically, the tumor was composed of mixed epithelial and stromal proliferation. (A) The epithelial components are arranged in a tubular pattern against in a background of ovarian-like stromal proliferation (HE stain, $\times 40$ ). (B) A single layer of cuboidal cells in the tubules with no significant cytologic atypia or mitosis, and with the mesenchymal components, which were composed of spindle cells that are arranged distributed from loose to dense (HE stain, $\times 200)$.
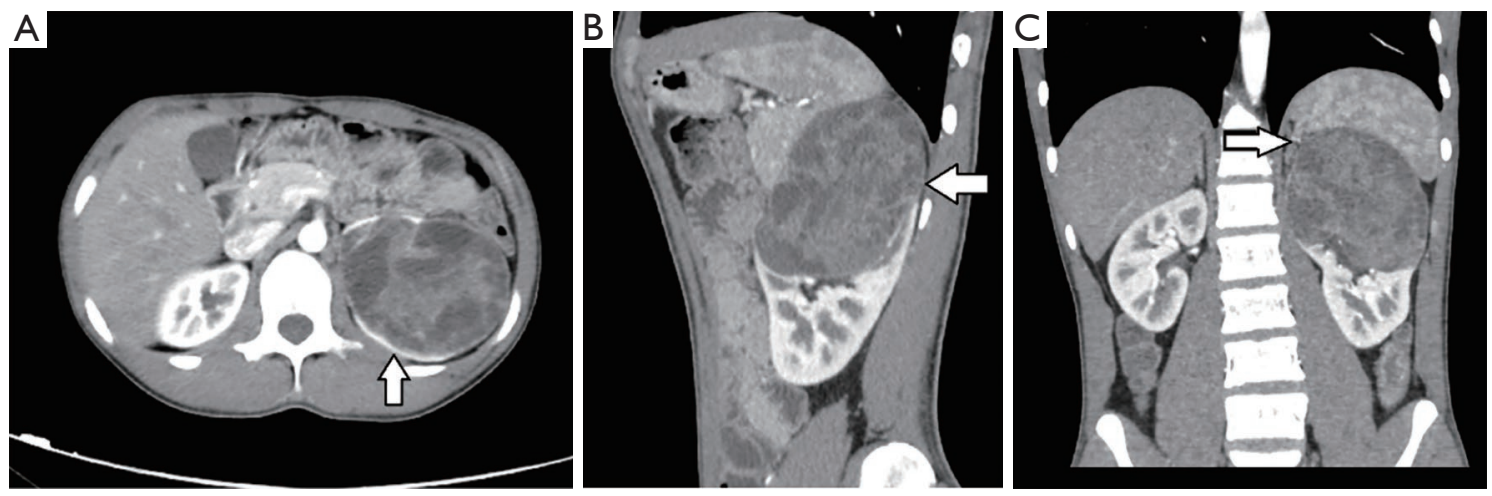

Figure 3 Abdominal corticomedullary CT showed that the tumor had heterogeneous soft tissue (white arrow). (A) In cross-sectional axial imaging; (B) in sagittal imaging; (C) in coronal imaging. CT, computed tomography.

palpation was completely normal. His presurgical blood investigations were within normal limits. The abdominal contrast-enhanced CT revealed an $8.2 \mathrm{~cm} \times 9.0 \mathrm{~cm}$ $\times 11.2 \mathrm{~cm}$ well-defined, uneven mass lesion of the left kidney. The solid component showed mild to moderate enhancement, which increased over time (Figure 3). There was also a $1.2 \mathrm{~cm} \times 1.0 \mathrm{~cm}$ well-defined nodule in the left adrenal gland, with enhancement, which increased over time. Magnetic resonance imaging (MRI) showed a $7.7 \mathrm{~cm}$ $\times 9.9 \mathrm{~cm} \times 9.6 \mathrm{~cm}$ cystic lesion that arose from the upper pole of the left kidney, with an uneven enhancement that increased over time. The patient was diagnosed with a malignant tumor of the kidney before the operation, and a left radical nephrectomy was performed. Subsequent surgical pathology revealed MESTK. Macroscopically, the tumor measured $10 \mathrm{~cm} \times 10 \mathrm{~cm}$ and was located at the upper pole of the left kidney. Both multiloculated cystic structures and solid components were revealed in the tumor cross-section.

Microscopically, the tumor was composed of mixed epithelial and stromal proliferation (Figure 4). Immunohistochemically some epithelial cells revealed the positive expression of CK7, CK. The stroma of tumor showed positive expression of SMA, desmin and vimentin. Some spindle cells showed expression of PR. There was no expression of ER, S-100 and CD34.The proportion of cells that expressed Ki-67 was $2 \%$. The final diagnosis was MESTK. Next generation sequencing showed a mutation in the $B R A F$ gene, which is a non-frameshift mutation. The patient was discharged without adverse outcomes. One month after the operation, no surgical complications were 

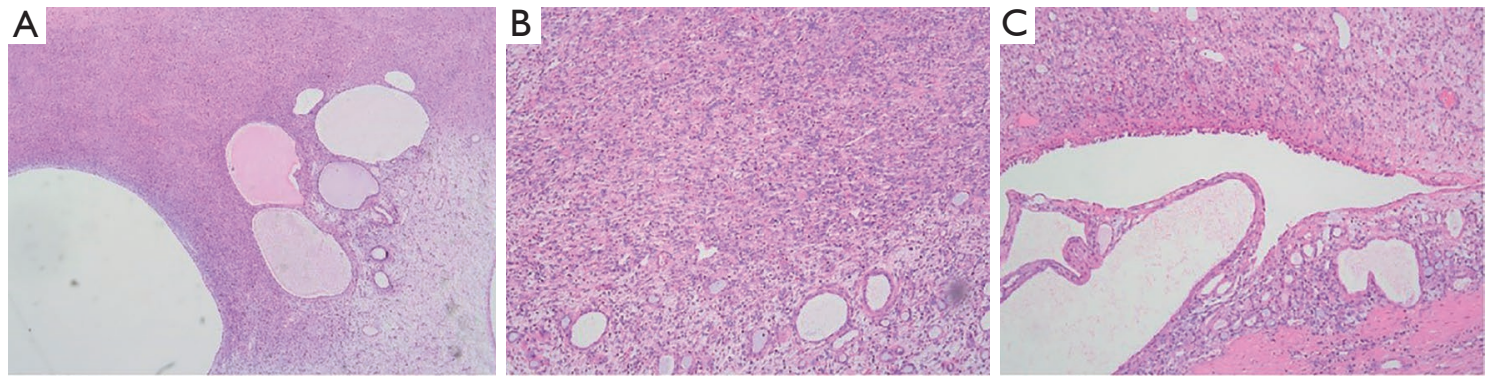

Figure 4 Microscopically, the tumor was composed of mixed epithelial and stromal proliferation. (A,B) The epithelial components were are arranged in a tubular and cystic pattern against in a background of ovarian-like stromal proliferation (HE stain, $\times 40)$. (C) The bland flattened cells or hobnail cells around the cystic component (HE stain, $\times 200)$.

observed. The patient was periodically monitored for one year following surgery, and there were no imaging findings of recurrence or metastasis.

\section{Ethical statement}

All procedures performed in studies involving human participants were in accordance with the ethical standards of the institutional and/or national research committee (s) and with the Helsinki Declaration (as revised in 2013). Written informed consent was obtained from the patient.

\section{Discussion}

Mixed epithelial and stromal tumors of the kidney is rare renal neoplasm which usually behaves benignly, but rarely malignancies have also been reported. Adult mesoblastic nephroma $(\mathrm{MN})$, multilocular renal cyst with müllerianlike stroma, solid and cystic biphasic tumor and cystic hamartoma of the renal pelvis have also been described. In 2004, the World Health Organization (WHO) published the Renal Tumor Classification. MESTK has been included in this classification. Zhou et al. reported that adult cystic nephroma $(\mathrm{CN})$ and MESTK share similar features in term of affected patients' ages, clinicopathologic characteristics, and immunohistochemistry (3). In 2016 the WHO Classification of Tumors of the Urinary System and Male Genital Organs, adult CN and MESTK were included in the category "mixed epithelial and stromal tumor family of the kidney" (4). Patients mainly present asymptomatically, but they can also present with flank pain, palpable abdominal masses, and hematuria; however, these clinical manifestations are not sufficiently specific to diagnose MESTK. With the popularity of routine health screening and development in imaging modalities, approximately $25 \%$ of MESTK are incidentally diagnosed $(5,6)$. Radiographically, MESTK appears as a multilocular cystic mass with a variable cystic component, which belongs to Bosniak Categories III to IV (5). CT is regarded as a diagnostic imaging tool for diagnosing MESTK. The classic CT presentation of MESTK is a centrally located, welldefined multiseptated cystic solid mass with thick or thin separations (7). The density of the cystic mass is uniform, and hemorrhage is rare, while delayed enhancement of the solid component during the nephrographic phase in the contrast-enhanced CT is also frequently observed $(8,9)$. Based on a blinded, retrospective review of the images of 6 cases of MESTK and 14 cases of cystic renal cell carcinoma (CRCC) on multi-slice computed tomography (MSCT), Lang et al. concluded that there are certain difficulties in making an accurate diagnosis preoperatively to differentiate MESTK and CRCC. However, the proportion of solid components, the characteristic septa, and the contrast enhancement pattern can provide helpful information (8). MESTK is often misdiagnosed as CRCC preoperatively.

Confirmation of histological features and IHC staining of tumors are the gold standards for tumor diagnosis. During the preoperative diagnosis of MESTK, it is difficult to distinguish it from other kidney tumors, especially CRCC. The amount of fine-needle-aspirated tissue of renal cystic lesions is too little to make an accurate diagnosis. Because of the risk of malignant cell expansion, fine-needle aspiration is not recommended in renal cystic lesions (10).

IHC staining of epithelial components and stromal components of MESTK demonstrate s different characteristics. The epithelial components are positive for PAX 8 and CK, especially CK7. The stromal components are positive for 
SMA, desmin, and vimentin. CD10 and inhibin can be positive in the stroma in some cases. The stromal components are negative for HMB-45, S-100, and melan-A (11-13).

MESTK must be differentiated from other renal cystic tumors. $\mathrm{CN}$ is predominantly cystic tumors composed entirely of differentiated tissues without solid components. CNs are strongly associated with mutations in the DICER1 gene (14). Cystic partially differentiated nephroblastoma (CPDN) consists of predominantly cystic and solid lesions, in which blastemal or other embryonal cells are present in the septa of the cysts. CPDN predominantly affect infants. Angiomyolipoma with epithelial cysts (AMLEC) also displays mixed, solid, and cystic architecture, which is composed of smooth-muscle-predominant (or "fatpoor") angiomyolipoma and epithelial cysts (14). However, AMLEC is positive for HMB-45 and melan-A, which is different from MESTK in IHC staining (15). Metanephric adenofibroma is also composed of both mesenchymal components and epithelial components, and it appears to affect predominantly young patients. Its epithelial component may show different subtypes, and the spindled stromal component features angiodysplasia, which is different from MESTK (16).

Due to the difficulty in preoperative diagnosis of MESTK, surgical treatment is the standard treatment of MESTK. In general, tumor rupture during surgery may be associated with a poorer prognosis. One case has been reported where a tumor ruptured intraoperatively, and local recurrence of the tumor occurred during the postoperative follow-up (17). One case has been reported where a tumor ruptured intraoperatively, and local recurrence of the tumor occurred during the postoperative follow-up (18). Renal cyst marsupialization is not recommended. Complete resection of the tumor by partial or complete nephrectomy is recommended according to the patient's condition.

Moreover, Minoda et al. reported a patient who was diagnosed as having bilateral MESTK and underwent a left partial nephrectomy only and active surveillance of the right lesion (2). Our report shows that next generation sequencing cannot help guide further treatment. However, the role of generation sequencing in MESTK requires further study. Some patients whose pathology displays malignant features may undergo additional treatment with chemotherapy and/or radiation. However, it is still unclear whether such treatment is beneficial. Nevertheless, the prognosis of MESTK is good. Malignant potential of MESTK has been reported $(19,20)$, and a patient with MESTK should be advised to receive continued to follow- up postoperatively.

\section{Conclusions}

MESTK is a rare renal neoplasm, which usually behaves benignly and is difficult to diagnose preoperatively. To improve the current understanding of these tumors, comprehensive studies on their pathogenesis and preoperative diagnosis are needed.

\section{Acknowledgments}

Funding: This study was supported by the National Natural Science Foundation of China (Grant No. 81970661 to BS), the Tai Shan Scholar Foundation (ts201511092 to BS).

\section{Footnote}

Reporting Checklist: The authors have completed the CARE reporting checklist. Available at https://dx.doi. org/10.21037/tcr-21-308

Conflicts of Interest: All authors have completed the ICMJE uniform disclosure form (available at https://dx.doi. org/10.21037/tcr-21-308). The authors have no conflicts of interest to declare.

Ethical Statement: The authors are accountable for all aspects of the work in ensuring that questions related to the accuracy or integrity of any part of the work are appropriately investigated and resolved. All procedures performed in studies involving human participants were in accordance with the ethical standards of the institutional and/or national research committee(s) and with the Helsinki Declaration (as revised in 2013). Written informed consent was obtained from the patient for publication of this case report and accompanying images. A copy of the written consent is available for review by the editorial office of this journal.

Open Access Statement: This is an Open Access article distributed in accordance with the Creative Commons Attribution-NonCommercial-NoDerivs 4.0 International License (CC BY-NC-ND 4.0), which permits the noncommercial replication and distribution of the article with the strict proviso that no changes or edits are made and the original work is properly cited (including links to both the formal publication through the relevant DOI and the license). See: https://creativecommons.org/licenses/by-nc-nd/4.0/. 


\section{References}

1. Michal M, Syrucek M. Benign mixed epithelial and stromal tumor of the kidney. Pathol Res Pract 1998;194:445-8.

2. Minoda R, Takagi T, Toda N, et al. Bilateral and multiple mixed epithelial and stromal tumors of the kidney: A case report. Mol Clin Oncol 2017;7:1005-7.

3. Zhou M, Kort E, Hoekstra P, et al. Adult cystic nephroma and mixed epithelial and stromal tumor of the kidney are the same disease entity: molecular and histologic evidence. Am J Surg Pathol 2009;33:72-80.

4. Humphrey PA, Moch H, Cubilla AL, et al. The 2016 WHO Classification of Tumours of the Urinary System and Male Genital Organs-Part B: Prostate and Bladder Tumours. Eur Urol 2016;70:106-19.

5. Wang CJ, Lin YW, Xiang H, et al. Mixed epithelial and stromal tumor of the kidney: report of eight cases and literature review. World J Surg Oncol 2013;11:207.

6. Tsai SH, Wang JH, Lai YC, et al. Clinical-radiologic correlation of mixed epithelial and stromal tumor of the kidneys: Cases analysis. J Chin Med Assoc 2016;79:554-8.

7. Picken MM, Bova D, Pins MR, et al. Mixed Epithelial and Stromal Tumor of the Kidney with Extension into Inferior Vena Cava: Case Report and Discussion of Adult Biphasic Cystic Renal Lesions and the Significance of Vascular Involvement. Case Rep Pathol 2018;2018:8234295.

8. Lang N, Liu J, Yang Y, et al. Differential Diagnosis of Mixed Epithelial and Stromal Tumor of Kidney and Cystic Renal Cell Carcinoma with Multi-slice CT. Chinese Journal of Medical Imaging 2012;20:729-33.

9. Ye J, Xu Q, Zheng J, et al. Imaging of mixed epithelial and stromal tumor of the kidney: A case report and review of the literature. World J Clin Cases 2019;7:2580-6.

10. Ljungberg B, Bensalah K, Canfield S, et al. EAU guidelines on renal cell carcinoma: 2014 update. Eur Urol
2015;67:913-24.

11. Caliò A, Eble JN, Grignon DJ, et al. Mixed Epithelial and Stromal Tumor of the Kidney: A Clinicopathologic Study of 53 Cases. Am J Surg Pathol 2016;40:1538-49.

12. Michal M. Benign mixed epithelial and stromal tumor of the kidney. Pathol Res Pract 2000;196:275-6.

13. Moslemi MK. Mixed epithelial and stromal tumor of the kidney or adult mesoblastic nephroma: an update. Urol J 2010;7:141-7.

14. van Peer SE, Pleijte CJH, de Krijger RR, et al. Clinical and Molecular Characteristics and Outcome of Cystic Partially Differentiated Nephroblastoma and Cystic Nephroma: A Narrative Review of the Literature. Cancers (Basel) 2021;13:997.

15. Zhang X, He XL, Zhao M. Renal angiomyolipoma with epithelial cysts: a clinicopathological analysis of four cases. Zhonghua Bing Li Xue Za Zhi 2020;49:244-9.

16. Yao DW, Qu F, Hu SW, et al. Metanephric adenofibroma in a 10-year-old boy: report of a case and review of the literature. Int J Clin Exp Pathol 2015;8:3250-6.

17. Yap YS, Coleman M, Olver I. Aggressive mixed epithelialstromal tumour of the kidney treated with chemotherapy and radiotherapy. Lancet Oncol 2004;5:747-9.

18. Farias JA, Laryea J, Gokden N, et al. Peritoneal seeding following incomplete resection of mixed epithelial stromal tumor of the kidney: First case report. Urol Ann 2016;8:114-7.

19. Svec A, Hes O, Michal M, et al. Malignant mixed epithelial and stromal tumor of the kidney. Virchows Arch 2001;439:700-2.

20. Jung SJ, Shen SS, Tran T, et al. Mixed epithelial and stromal tumor of kidney with malignant transformation: report of two cases and review of literature. Hum Pathol 2008;39:463-8.
Cite this article as: Huang H, Jiang X, Shi B, Yin G, Cui J, Guo C, Yu C, Meng H. Case report: two rare cases of mixed epithelial and stromal tumor of the kidney and a review of the literature. Transl Cancer Res 2021;10(9):4256-4261. doi: 10.21037/ tcr-21-308 